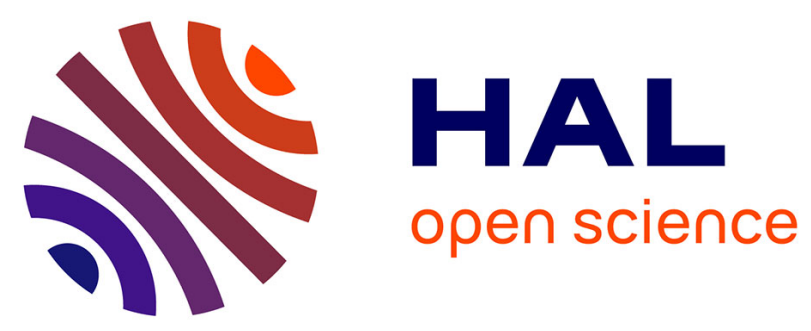

\title{
Reconstruction of angular speed variations in the angular domain to diagnose and quantify taper roller bearing outer race fault
}

\author{
Adeline Bourdon, Simon Chesné, Hugo André, Didier Rémond
}

\section{To cite this version:}

Adeline Bourdon, Simon Chesné, Hugo André, Didier Rémond. Reconstruction of angular speed variations in the angular domain to diagnose and quantify taper roller bearing outer race fault. Mechanical Systems and Signal Processing, 2019, 120, pp.1 - 15. 10.1016/j.ymssp.2018.09.040 . hal-01903288

\section{HAL Id: hal-01903288 \\ https://hal.science/hal-01903288}

Submitted on 19 Nov 2018

HAL is a multi-disciplinary open access archive for the deposit and dissemination of scientific research documents, whether they are published or not. The documents may come from teaching and research institutions in France or abroad, or from public or private research centers.
L'archive ouverte pluridisciplinaire HAL, est destinée au dépôt et à la diffusion de documents scientifiques de niveau recherche, publiés ou non, émanant des établissements d'enseignement et de recherche français ou étrangers, des laboratoires publics ou privés. 


\title{
Reconstruction of angular speed variations in the angular domain to diagnose and quantify taper roller bearing outer race fault
}

\author{
Adeline Bourdon ${ }^{\mathrm{a}}$, Simon Chesné ${ }^{\mathrm{a}}$, Hugo André ${ }^{\mathrm{b}}$, Didier Rémond ${ }^{\mathrm{a}, *}$ \\ a Univ Lyon, INSA-Lyon, CNRS UMR5259, LaMCoS, F-69621, France \\ ${ }^{\mathrm{b}}$ Université de Lyon, UJM Saint-Etienne, LASPI, F-42334, France
}

\section{A R T I C L E I N F O}

\section{Article history:}

Received 22 December 2017

Received in revised form 28 September 2018

Accepted 29 September 2018

\section{Keywords:}

Bearing fault

Non stationary conditions

Rotating machines

Instantaneous Angular Speed

Angular sampling

\begin{abstract}
A B S T R A C T
Monitoring of rotating machines is usually performed on vibration signals and associated signal processing tools focus on extracting weak cyclic information induced by faults on mechanical components like bearings. Their sensitivity to operating conditions in speed and torque are of huge interest in recent research activities. An alternative signal consists in measuring Instantaneous Angular Speed (IAS) of the machine which has been empirically demonstrated as a good vector of information. Angularly sampled by nature, this signal investigates other transfer paths from fault to transducer and introduces new challenges in understanding the relationships between operating conditions and effects of faults on the rotating speed of the machine. The main contribution of this paper lies on the introduction of basic signal processing techniques in order to analyze the shape of rotating speed fluctuations introduced by bearing faults. Modifications due to operating conditions and size of the defect are clearly separated in our suggested analysis on IAS. Another point of interest relies on the fact that this tool can be used in stationary or non-stationary operating conditions. All the analysis are given on experimental data performed on a specific test bench with different artificial bearing faults in various operating conditions.
\end{abstract}

(c) 2018 Elsevier Ltd. All rights reserved.

\section{Introduction}

Nowadays, monitoring of rotating machines faces the absolute need to be available and fully functional during nonstationary conditions, particularly in wind energy production. Traditional monitoring means are based on vibration signal analysis [1] while some emerging techniques use other signals like acoustic emission [2] or Instantaneous Angular Speed (IAS) [3]. The use of Instantaneous Angular Speed in rotating machine monitoring mainly relies just on the frequency content analysis of the IAS signal. This original approach has already been shown able to detect bearing defects on an industrial test case [4]. However, this empirical observation has not been yet clearly explained by specific measurement and some recent works are focusing on emerging modelling [5]. This lack of understanding can be justified by the following two limitations. First, usual mechanical models are confined to radial vibration influences of a bearing fault [6] and associated signal processing tools $[7,8]$. On the other hand, the frequency analysis of IAS shows the existence of bearing harmonics but the shape and

\footnotetext{
* Corresponding author.

E-mail addresses: adeline.bourdon@insa-lyon.fr (A. Bourdon), simon.chesne@insa-lyon.fr (S. Chesné), hugo.andre@univ-st-etienne.fr (H. André), didier. remond@insa-lyon.fr (D. Rémond).
} 
the magnitude of speed variations associated with these harmonics have never been quantified on the IAS. Whether the signal is obtained using demodulation technique [9] or elapse time method [10], it is always analyzed with conventional spectral observation tools [11]. These techniques, widely known and optimized for the analysis of traditional vibration signals, will be shown in this paper to be inadequate with IAS when applied on a bearing outer ring defect since they are not able to distinguish the healthy from the defective behavior if the operating conditions change.

Our alternative proposition is based on the fact that measuring rotating speed delivers another signal with different kind of information. Experimental evidence of bearing defect effects on Instantaneous Angular Speed has been proposed by several authors since two decades on different devices [12]. Moreover this alternative signal takes advantage of a different transfer path from excitation to response of the rotating machine. Another important feature lies on the fact that IAS measurements induce implicitly angular sampling and allow very long duration measurement without disturbances from nonconstant operating speed of the machine. This is the general context of the present paper. The objective is to answer questions arising from this framework in rotating machine where signal processing tools as well as phenomena have to be rethought from their basics.

The experimental evidence that will be shown in this paper support predictions made in [13], whereby the always varying load distribution inside the healthy bearing intrinsically induces speed variations of the cage and the shaft. As a consequence, this paper proposes an approach simply based on the angular observation of the IAS signal rather than on its spectral observation, and will therefore add grist to our mill concerning the following questions:

- How to detect the presence of a fault in the IAS signal?

- How to measure a fault severity in the IAS signal?

The two questions will be both focused on industrial issues. Answers will also help in submitting mechanical and phenomenological models to simulate correctly the bearing fault influence over the angular speed behavior of the whole mechanism.

The aim of the tool proposed in this paper is the use of the reconstruction of IAS variations signal in order to detect the presence of a fault on a bearing and to estimate its size. Another interest is to characterize the shape of induced fluctuations of IAS in order to state if there are consistent with physical phenomena. This tool is firstly presented in the general case and then applied to the actual case of an outer ring fault on a laboratory test rig. Several bearings, healthy or presenting various severity degrees of fault, will be compared. The test rig used in this study has been specially realized to ease experimentations on IAS and the reconstruction of IAS signal robustness will be shown for different operating conditions (radial load, speed, torque). Preliminary works have been published beforehand [21]. They show the feasibility and potential interest of the proposed tool but analyses were not sufficiently advanced, especially the actual size of the defects was overestimated. Moreover, analyses are also performed on healthy bearings. These new analyses are useful to identify the entrance and exit of a rolling element on a defect. Influences of bearing frequency slipping during measurement are also presented.

\subsection{General background}

This work is part of the broader framework of monitoring and maintaining rotating machinery and more specifically diagnosing localized faults in bearings leading to small variations of the dynamic behaviour. One challenge is to develop tools able to detect these variations and then to analyse their results to obtain information about the fault [14]. The vast majority of these works concerns the study of accelerometer signals and the use of time or frequency analyses. Recent research activities are also concerned with time-frequency analysis or by tools related to cyclostationarity approach [15]. The main tendency consists in providing new signal processing tools applied on traditional accelerometer signals. Huge scientist efforts are engaged to tackle sensitivity of these processing tools to operating conditions in speed or in load. For that, artificial intelligence approaches have been intensively investigated in order to take into account the large amount of information contained in vibration signals but not necessarily revealed by a single processing tool. Vectors of many different indicators are then processed by heuristic methods to organise and to extract the most significant information related to the health state of the machine under monitoring.

Based on this background, the proposed approach is mainly devoted to the understanding of phenomena involved in IAS variations. The main objectives are to propose a signal processing tool in order to characterise the components in IAS signal attached to tapered roller bearing defects. This approach allows to separate the operating condition effects, mainly associated with the magnitude of IAS variations, from the fault size effect. To estimate the contribution of this approach the experimental signals measured on a test bench are analysed using a global spectral indicator and by our alternative method. The tool proposed herein appears as a good candidate to detect an outer race bearing fault and to estimate its length and to understand the link between defect geometry and its manifestation on IAS signal.

\subsection{Characteristic frequencies and angular sampling}

As said earlier, the experimental work carried out on different mechanical systems and in various operating conditions have shown that the presence of a localized defect on a gear tooth or a bearing raceway will lead to weak changes in the dynamic behaviour of the mechanical system. Resulting disturbances are of short time duration and appear cyclically. Let 
$A_{\theta}(r e v)$ be this period and $f_{\theta}(e v / r e v)$ the corresponding angle frequency. $A_{\theta}$ and $f_{\theta}$ only depend on the geometry of the component of interest and are called, respectively, characteristic period and frequency. Gear characteristic frequency is the meshing frequency and depends only on the number of gear teeth. Bearing characteristic frequencies refer either to Ball Pass Frequency on the Outer ring (BPFO), to Ball Pass Frequency on the Inner ring (BPFI) or to the Fundamental Train Frequency (FTF). All these characteristic frequencies have their corresponding orders in the order frequency domain (BPOO for Ball Pass Order on the Outer ring, BPOI for Ball Pass Order on the Inner ring and FTO for Fundamental Train Order). The calculation of these frequencies requires the precise knowledge of the internal geometry of the bearing which is usually not shared by manufacturers. But they provide nominal values for these frequencies. However, it should be noticed that characteristic nominal frequencies are calculated for theoretical operating conditions including no slip assumption and without taking into account varying parameters like temperature or actual contact angle. In many applications, they can vary from $1 \%$ to $2 \%$ around their nominal value. As most of the diagnostic techniques the monitoring tool proposed in this paper is based on these characteristic frequencies and considers that, despite these small variations, these bearing frequencies can be localized and identified in experimental signal measurement.

If the machine rotates at a constant speed $\Omega\left(\mathrm{rad} \cdot \mathrm{s}^{-1}\right)$ the relation between shaft angular position $\theta(\mathrm{rad})$ and time $\mathrm{t}(\mathrm{s})$ is:

$$
\theta=\Omega . t
$$

Therefore it is possible to define the time period between two defect events as $\mathrm{T}$ in the following way:

$$
A_{\theta} .2 \pi=\Omega . T
$$

Note that bearing characteristic frequencies are commonly given for a specific constant rotating speed $\Omega_{0}\left(\mathrm{rad} \cdot \mathrm{s}^{-1}\right.$ ) and expressed in Hertz. The relationship between a characteristic frequency $\mathrm{f}_{\mathrm{Hz}}$ and its associated angular periodicity (or cyclic periodicity) is:

$$
f_{\mathrm{Hz}}=\frac{1}{T}=\frac{\Omega_{o}}{A_{\theta} \cdot 2 \pi}=\frac{\Omega_{o}}{2 \pi} f_{\theta}
$$

In case of non-stationary speed condition Eq. (1) becomes:

$$
\theta(t)=\int_{0}^{t} \Omega(u) \cdot d u
$$

Time between two angularly periodic events is not constant anymore. The use of the angular approach is then essential to keep a steady definition of frequencies expressed as generalized orders in reference with the shaft revolution.

In the angular approach, measured signals are sampled with a constant angle step rather than with a constant time step. Angular sampling can be achieved directly through the acquisition process [16] or through post processing of time sampled signals using classical interpolation procedures [17]. All these angular approaches are now very well-known [18]. Usual techniques of signal processing may be applied on these angular signals such as transformation in the frequency domain using the Fourier transform. The spectra are then expressed in terms of angle frequency (or cyclic frequency). In such spectra the characteristic frequencies of the rotating parts are always well localized regardless the operating macroscopic speed of the machine. The contribution of a specific part of the rotating machine will be concentrated on a particular and unique channel in the frequency analysis regardless the speed of the machine. Angular sampling allows long measurements where low energy components may be emphasized on a single channel in a high resolution spectrum [19]. This specific property will be the core basis in the following approach.

\subsection{Instantaneous Angular Speed used for monitoring rotating system}

Instantaneous Angular Speed signals used in this paper to monitor bearing defects are obtained by the elapsed-time method which has been the subject of numerous publications [9-11]. It is based on the simultaneous use of an encoder with a resolution of $R$ impulses per revolution and of a high-frequency $f_{c}$ counting clock. It consists in counting the number $n_{c i}$ of clock ticks between two successive pulses of the encoder signal. This first signal $\left(\theta_{\mathrm{i}}, \mathrm{n}_{\mathrm{ci}}\right)$ is by construction sampled in the angular domain and allows easy access to the angle-time relationship:

$$
t_{i}=t\left(\theta_{i}\right)=\frac{1}{f_{c}} \cdot \sum_{j=1}^{i} n_{c j}
$$

It also allows to estimate the average speed $\bar{\omega}_{i}$ for each angular step $\Delta \theta_{\mathrm{i}}$ by Eq. (6), this signal being called Instantaneous Angular Speed:

$$
\bar{\omega}_{i}=\frac{\Delta \theta}{\Delta t}=\frac{2 \pi}{R} \cdot \frac{f_{c}}{n_{c i}}
$$

This method allows high precision estimation of IAS [19] on long measurements and then a high resolution in spectral analysis. Several authors have demonstrated the effectiveness of IAS signals [9] and used them in various applications [9 -12 ] but usually in a spectral analysis. Usual techniques of signal processing can be applied to IAS which appears very useful 
for monitoring and diagnosing different faults in rotating components such as bearing outer ring defects [9,12]. These technics allow to detect the presence of a faulty bearing but do not give information on the shape and magnitude of the speed variations induced by a bearing. Indeed, most of the usual monitoring tools have been developed for acceleration signals and stationary operating conditions. These signals do not have very low frequency components and information on the magnitude of the disturbance can be estimated. On the opposite, IAS signal is mainly composed of macroscopic fluctuations due to operating speed conditions and components of interest in low frequency which are very difficult to exhibit.

Moreover, measurements performed on different devices show that in some operating conditions, BPOO can be detected in IAS spectrum for healthy bearings. Therefore, detection of the BPOO in the IAS spectrum is not enough to guarantee the presence of a defect. It appears necessary to have information on the shape and magnitude of speed variations due to the natural bearing rotation and to estimate the influence of the defect on them.

\section{Reconstitution of angular speed variations using inverse Fourier transforms}

\subsection{Principle}

Assuming that the IAS signal $\omega$ depending on the angular position $\theta$ can be written as

$$
\omega(\theta)=\omega_{b}(\theta)+\tilde{\omega}(\theta)
$$

where $\omega_{\mathrm{b}}$ gathers speed components associated with the considered bearing BPOO and $\tilde{\omega}$ is the residual speed. The proposed tool is able to extract $\omega_{\mathrm{b}}$ from $\omega_{\text {. }} \omega_{\mathrm{b}}$ has by definition a constant angular period, which is translated in the angle frequency domain by the emergence of peaks located at harmonics of the bearing of interest BPOO. Most of monitoring techniques are based on indicators computed using magnitude of these harmonics, the proposed approach is only an alternative way to understand how these harmonics are impacted by the defect and how they can be used to estimate the size of the defect.

The suggested signal processing tool is basically based on Fourier and inverse Fourier transforms. It consists on extracting from the angle frequency spectrum the harmonics associated with the bearing BPOO and then to come back in the "angle domain" using inverse Fourier transform. Then, it is possible to have information on the shape and magnitude of the speed variations $\omega_{\mathrm{b}}$ and to estimate the influence of a bearing defect.

The methodology of the proposed tool can be summarized as follow:

1. Measuring IAS signal $\omega$,

2. Computing its angle frequency spectrum without any normalization about the signal size,

3. Identifying the actual angle frequency $\mathrm{f}_{\theta}$ associated with bearing outer race (actual $\mathrm{BPOO}$ ),

4. Editing the angle frequency spectrum and selecting channels of interest by using $m$ rectangular windows centered on the first $m$ harmonics of the characteristic frequency $\mathrm{f}_{\theta}$. Details of this step are given in Section 2.2.

5. Reconstructing the filtered angular signal $\omega_{\mathrm{b}}$ in angular domain which represents IAS variations linked to the bearing characteristic angle frequency $\mathrm{f}_{\theta}$ identified in step3.

\subsection{Spectrum editing step}

The reconstruction is performed by employing $m$ rectangular windows centered on the first $m$ harmonics of the characteristic fault frequency. So the main step consists in identifying the characteristic fault frequency. But during measurement this frequency may vary of several percent around its nominal value due to slipping conditions or weak variations in operating conditions. The proposed tool can be used only when $\mathrm{f}_{\theta}$ can be identified it is to say when a peak can be found in the magnitude spectrum of the IAS in the vicinity, defined by the user, of the nominal BPOO (green point in Fig. 1a). In the
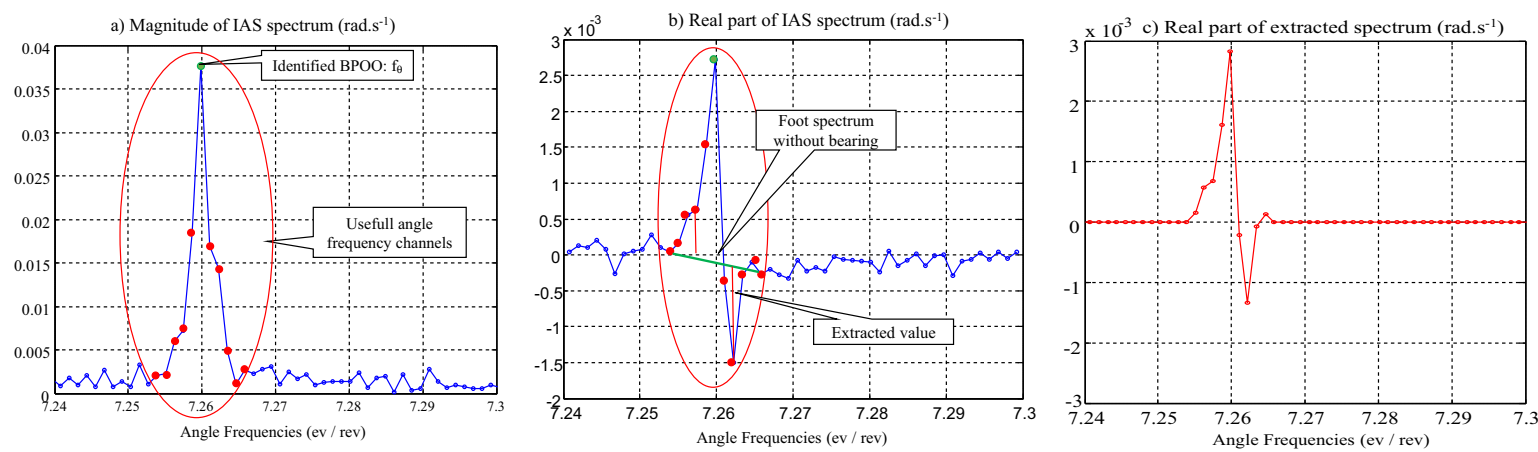

Fig. 1. Editing the angle frequency spectrum principle. 
present work, this identification is performed by the user but automation of this step is probably possible. Some further details about frequency slipping will be given in Sections 3.2 and 3.5. The width of the associated harmonics expresses that the cyclic frequency can slightly vary during measurement. This width is also identified by the user and defined as $2 n+1$ angle frequency channels centered on $\mathrm{f}_{\theta}$. In case presented in Fig. $1 \mathrm{a}, n$ equals 5.

Real part of the spectrum is shown in Fig. 1b. The values associated with the selected channels are considered (green and red points in Fig. 1b). Between the first and the last frequency channels, the foot of the undisturbed spectrum is assumed to be a straight line (green line in Fig. 1b). The value of each selected channel with regard to the spectrum foot is extracted, leading to the extracted real part spectrum shown in Fig. 1c. Same process is applied on the imaginary part of the spectrum.

This processing method is performed on $m$ harmonics of the BPOO. In practice $m$ corresponds to the number of harmonics with significant magnitude. Let's $n_{\theta}$ be the closest channel number corresponding to the event frequency $f_{\theta}$. The channels used for filtering information linked to the $j^{\text {th }}$ harmonic are between the $\left(j . n_{\theta}-n\right)$ th and $\left(j . n_{\theta}+n\right)$ th ones. By construction the mean value of the filtered signal is nil as there is no DC in the resulting filtered spectrum. Then the resulting filtered spectrum contains 2.m. $(2 n+1)$ components different from zero extracted from a large amount of components. In another hand more components are gathered in this filtered signal than the number of components used in classical indicators where only magnitudes of the first $m$ harmonics of the BPOO are summed. The number $m$ of useful harmonics and $n$ of useful channels are specific to the considered experimental data and have been chosen to build both smooth and representative waveform of IAS variations induced by healthy or faulty bearings. Without any further indication, in the present work $n$ and $m$ are set to 10 .

Fig. 2 illustrates these different steps for one bearing during measurement M2 defined in Section 3. The macroscopic speed variation shown in Fig. 2a will be explained in Section 3.1 and analysis of the resulting IAS filtered variations shown in Fig. $2 \mathrm{~d}$ is the aim of the present paper.

a) Step 1 : Measuring IAS Signal

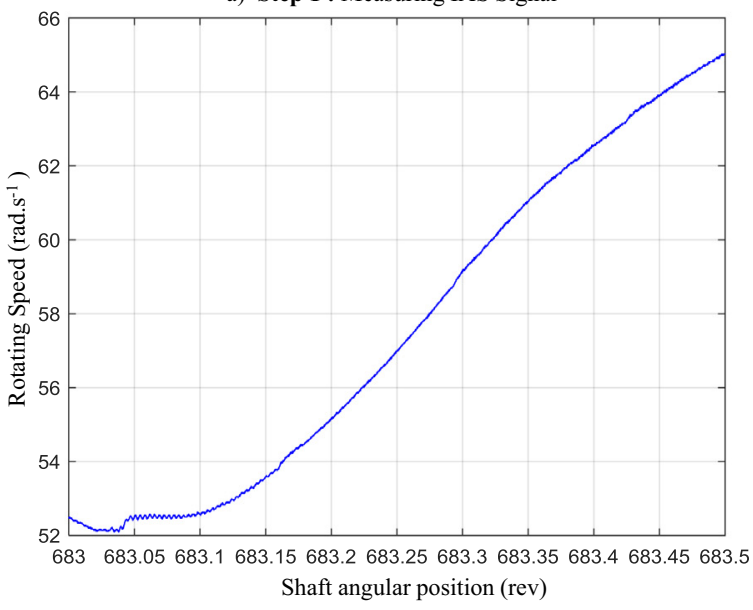

c) Step 3 : Extracting usefull channels

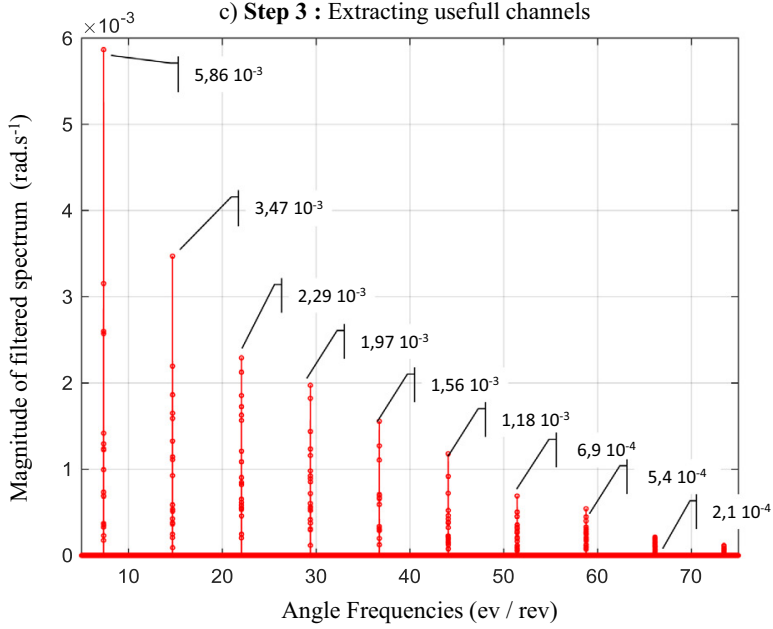

b) Step 2 : Computing IAS angle frequency Spectrum

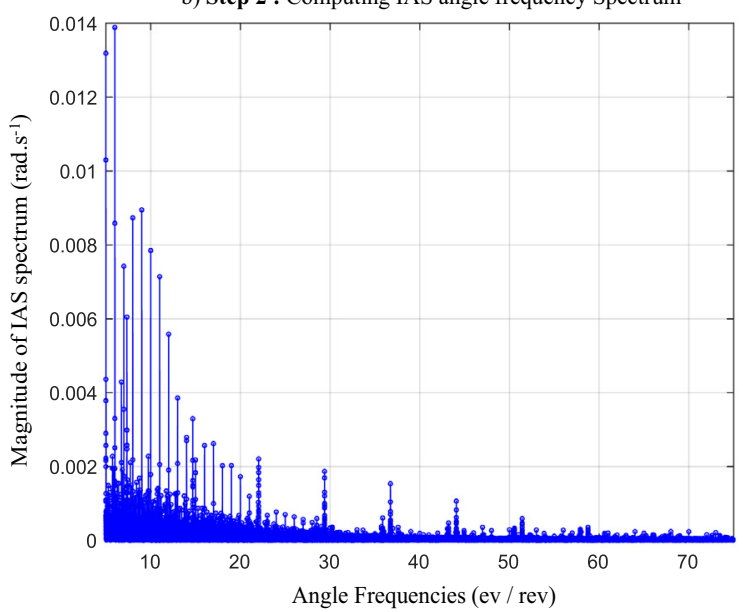

d) Step 4 : Computing filtered IAS variations

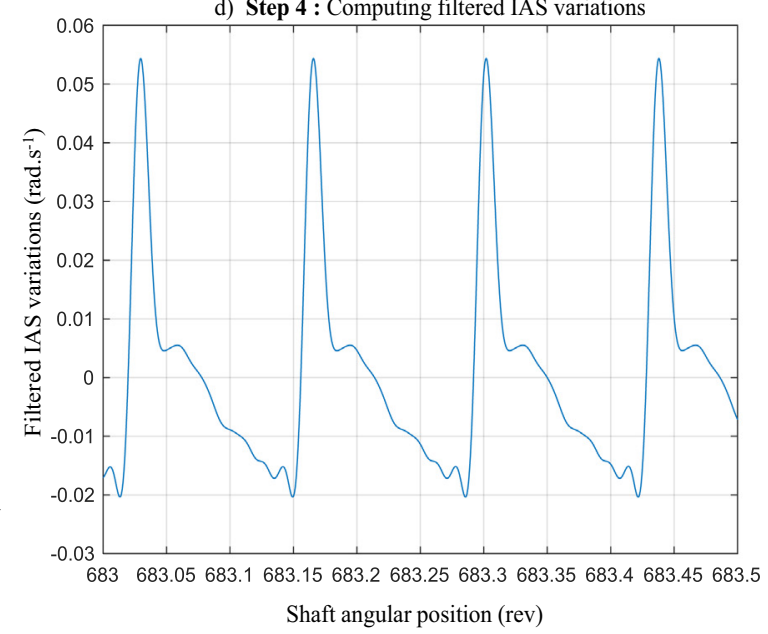

Fig. 2. Illustration of the 4 steps of the proposed tool. 


\section{Experimental applications}

In this section IAS signals, measured on a dedicated test bench described below, are analyzed for healthy and faulty bearings and for different operating conditions.

\subsection{Test bench description}

A specific test bench presented in Fig. 3 has been developed. It consists in a shaft supported by two identical tapered roller bearings (SNR $32005 \mathrm{~V}$ ) with $Z=17$ rolling elements and a nominal BPOO provided by SNR equals to $7.26 \mathrm{ev} \cdot \mathrm{rev}^{-1}$. The bearing 2 is always healthy while the bearing 1 can be healthy or presented different levels of spalling on its outer ring. Faults are artificial and obtained by electro erosion but care has been taken to have defects with realistic sizes and shapes. For the present analysis, three different sizes of defect are considered (Fig. 4). In practice only the outer ring of bearing 1 is replaced for each measurement. An original device composed of a spherical bearing and two springs allows to apply adjustable radial loads independently on the rotation speed. The position of this device can be adjusted along the shaft. In the present work, it is centered between the two bearings, so the radial loads on the two bearings are assumed to be the same. An axial preload can also be applied in the form of an imposed displacement of one bearing outer ring. A high accuracy optical encoder $(R=5000$ pulses per revolution), not shown in Fig. 3, coupled to an acquisition device with a high frequency clock at $\mathrm{fc}=80 \mathrm{MHz}$ ensures the angular sampling and measurement of the Instantaneous Angular Speed by the elapse time method. For more details on the advantage and limitation of this method see [20]. This shaft is placed in revolution by an electric motor via a flexible coupling.

This bench allows to test the influence of operating parameters (load, macroscopic velocity) on rotating speed variations and to compare, for a given operating condition, the effect of the bearing fault.

Due to motor control, despite a constant control voltage of the electrical motor $(3.2 \mathrm{~V})$ the rotating speed of the device may vary slightly as shown in Fig. 5a. Angle frequency analysis, Fig. 5b, shows that these variations are associated with phenomena whose angle frequencies are 1, 2, 3 and 4 events per revolution. So analysis presented in the following are not obtained for stationary rotating speed, but around a macroscopic mean speed.

Lengths $L_{d}$ of the defect which are measured along the rolling direction are given in Table 1 . These defects are localized on the outer raceway at a diameter $\mathrm{d}(40.5 \mathrm{~mm})$, so they correspond to an angle $\theta_{\mathrm{d}}$ defined by Eq. (8)

$$
\theta_{d}=\frac{2 . L_{d}}{d}
$$

Angle between two rolling elements is $\theta_{0}$ defined by Eq. (9) where $Z$ is the number of rolling elements.

$$
\theta_{0}=\frac{2 . \pi}{Z}
$$

To allow working with dimensionless data, angle ratio $r_{\theta}$ between the fault angle $\theta_{d}$ and the reference angle $\theta_{o}$ between two rolling elements, are also given in Table 1

$$
r_{\theta}=\frac{\theta_{d}}{\theta_{o}}
$$

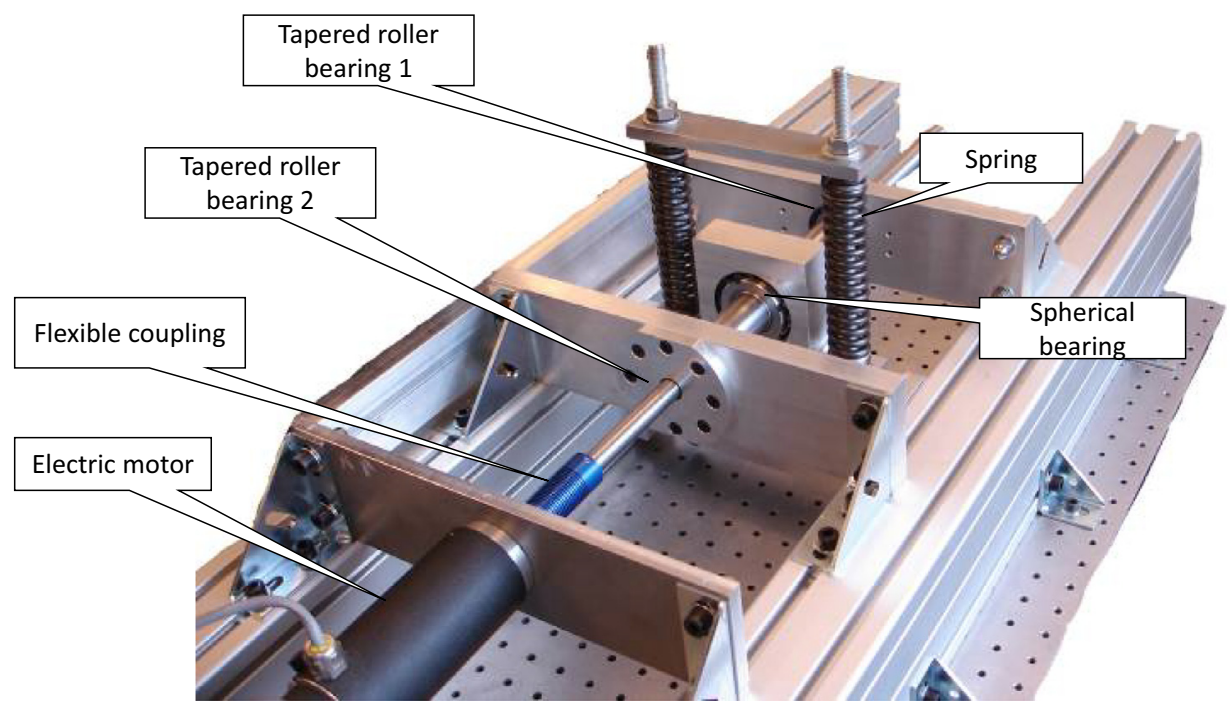

Fig. 3. Specific test bench based on a simple rotating shaft. 

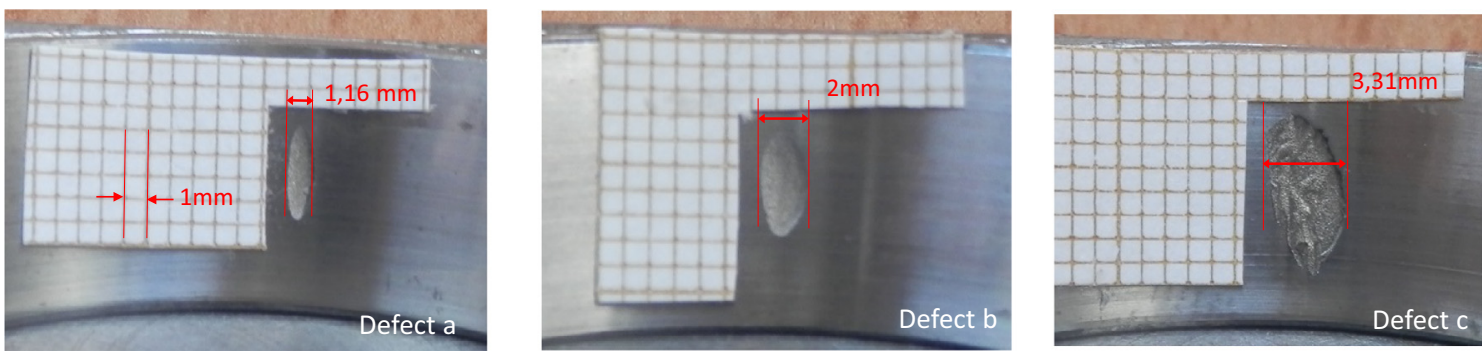

Fig. 4. Artificial bearing faults on outer race.

a) Macroscopique speed variations

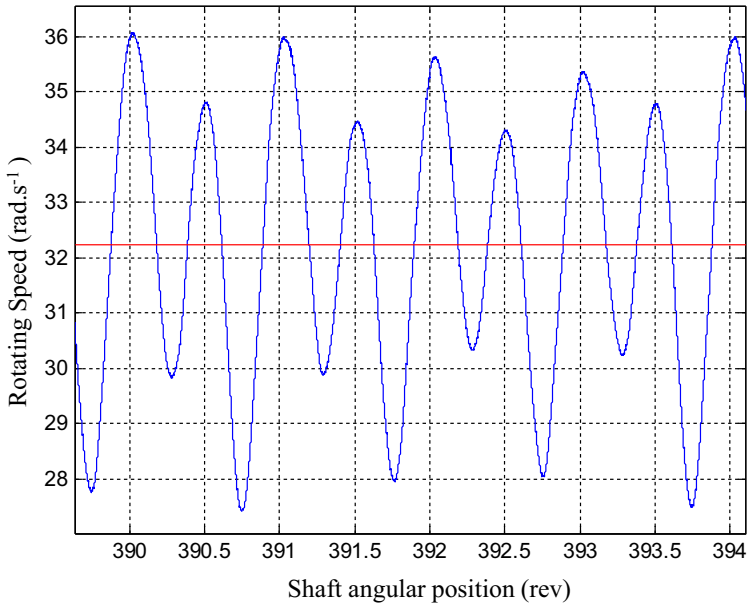

b) Speed variations spectrum

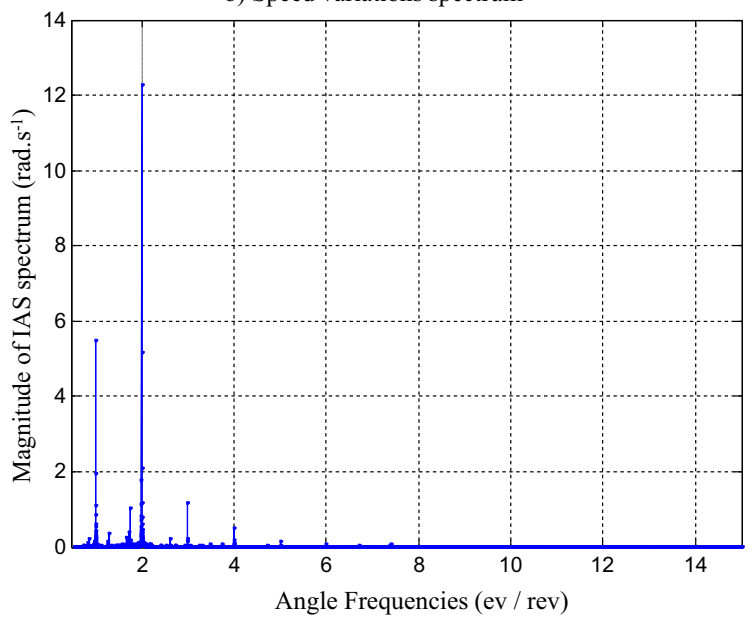

Fig. 5. Macroscopic speed variations and corresponding spectrum.

Table 1

Geometrical characteristics of defects.

\begin{tabular}{lll}
\hline Defect & Length $\mathrm{L}_{\mathrm{d}}(\mathrm{mm})$ & Angle ratio $\mathrm{r}_{\theta}$ \\
\hline a & 1.16 & 0.15 \\
b & 2 & 0.27 \\
c & 3.31 & 0.44 \\
\hline
\end{tabular}

As said earlier actual values of BPOO may vary of several percents around its nominal value due to slipping conditions or weak variations in operating conditions. Consequently even with similar operating conditions the two bearings of the test bench may have different actual BPOO. Taking advantages of long duration measurement and angular sampling, IAS measurement with elapse time method will give sufficient precision on spectral analysis to separate these weak differences in BPOO from the two bearings. Fig. 6 gives an illustration of these BPOO differences with two healthy bearings configuration in Fig. 6a. and with one healthy and one faulty bearing configuration in Fig. $6 \mathrm{~b}$. BPOO variations $\Delta$ f indicated in Fig. 6 is the relative variation from its nominal value $(7.29 \mathrm{ev} / \mathrm{rev})$ and the actual value identified in the angular spectrum. They are less than $2.5 \%$ for all the tested bearing associated to each component. So, for each measurement it is possible to have information from the two bearings but it is impossible to identify the bearings. In Fig. 6a. we are not able yet to explain precisely why the magnitude of the two peaks is different. The most relevant for us is related to the fact that the BPOO of bearing "B" presents a larger width which is associated to more fluctuations in bearing kinematics (see Section 3.5). Dispersions in measurement and influence of the overall architecture of the test bench can also explain this difference. By comparing the magnitude of the identified peaks in Fig. $6 \mathrm{~b}$ (corresponding to a healthy and a faulty bearings) with the magnitude of healthy bearings in Fig. 6 a (about $2.10^{-3} \mathrm{rad} \cdot \mathrm{s}^{-1}$ ), we can guess that the bearing associated with the peak $A$ is the faulty bearing but we are not able yet to locate it in bearing position 1 or 2 .

To estimate the contribution of the proposed approach, signals are analysed using global indicators called $\mathrm{H}_{\mathrm{sm}}$ and by the proposed method. $\mathrm{H}_{\mathrm{sm}}$ is defined as the sum of the maxima of each filtering window. For example for a bearing of measurement M2 the maxima of each filtering window is given in Fig. $2 \mathrm{c}$ and the corresponding $\mathrm{H}_{\mathrm{sm}}$ can be computed by: 

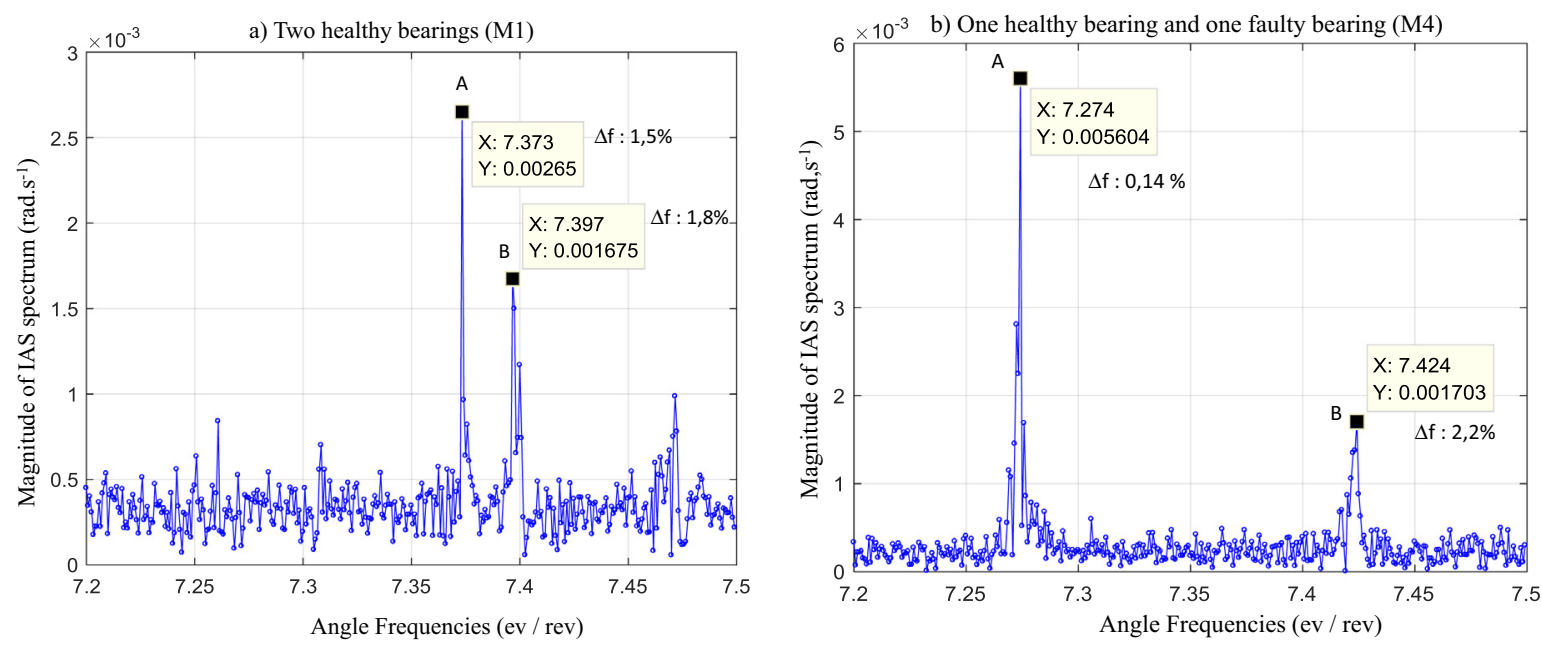

Fig. 6. Effective BPOO localization in IAS spectrum.

$$
H_{s m}=(5.86+3.47+2.29+1.97+1.56+1.18+0.69+0.54+0.21) \cdot 10^{-3}=0.017
$$

So for each measurement when two peaks appear clearly two indicators are computed. Operating conditions and $\mathrm{H}_{\mathrm{sm}}$ for the measurement analysis are summarized in Table 2. By convention $\mathrm{H}_{\mathrm{sm} 1}$ is assumed to be the higher, we will see in Section 3.2 that it can be related to the faulty bearing. All measurements described in this section consist in signals of 6 million points in length (i.e. 1200 shaft revolutions).

\subsection{Analysis of speed variations in case of healthy bearing}

As previously mentioned, existence of BPOO harmonics in the speed signal is not revealing the presence of a fault on the outer race. Indeed as demonstrated in [5] for deep groove ball bearings, BPOO components are also related to loading variations on rolling elements during the shaft rotation as illustrated in Fig. 7. The analysis suggested may be very helpful for estimating the shape of speed fluctuations due to healthy bearing.

For the two healthy bearings of measurement M1 their filtered speed variations are plot in blue in Fig. 8a. In order to compare phenomena with angle periods presenting weak differences, the horizontal axis is graduated in multiple of period $\mathrm{A}_{\theta}$ (1/BPOO) defined in Section 1.1 of the considered bearing and the origin of the vertical axis is arbitrarily fixed. The shape of these variations are very similar and close to a sinusoid. In the following we will consider that this shape of the filtered speed variation is the signature of a healthy bearing. For measurements M2 and M4 performed in the same operating conditions than M1 (mean speed of $60 \mathrm{rad} / \mathrm{s}$, radial load of $3200 \mathrm{~N}$ ) the filtered speed variations associated to the bearing with the lowest $\mathrm{H}_{\mathrm{sm}}$ factor are also plotted in Fig. 8a. They have similar signatures than those of the healthy bearings of measurement M1. So we can conclude that in case of bearings operated in the same conditions the value $H_{s m}$ allows to identify the healthy and faulty bearings. This conclusion can be strengthened by comparing measurement M2 in Figs. $8 \mathrm{a}$ and $2 \mathrm{~d}$. In Fig. $2 \mathrm{~d}$ filtered speed variations are plotted for the bearing associated to the higher $\mathrm{H}_{\mathrm{sm}}$ exhibiting a different shape from the healthy bearing.

Fig. $8 \mathrm{~b}$ shows the filtered IAS variations for the healthy bearing of measurements M3 and M4 which correspond to the same rotating speed (mean speed of $60 \mathrm{rad} / \mathrm{s}$ ) but different radial load ( $2200 \mathrm{~N}$ and $3200 \mathrm{~N}$ ). It shows that an increase of the radial load leads to an increase of the magnitude of the filtered IAS variations. This phenomenon is not significantly detectable in the global indicator as the associated $\mathrm{H}_{\mathrm{sm}}$ is 0.0024 for M3 and 0.0027 for M4.

Table 2

Measurement operating conditions and corresponding global indicator $\mathrm{H}_{\mathrm{sm}}$.

\begin{tabular}{lllll}
\hline Measurement & Bearings condition & Radial load $(\mathrm{N})$ & ${\text { Mean speed }\left(\mathrm{rad} \cdot \mathrm{s}^{-1}\right)}^{\mathrm{H}_{\mathrm{sm} 2}}$ \\
\hline M1 & Healthy - Healthy & 3200 & 60 & 0.0025 \\
M2 & Defect a - Healthy & 3200 & 60 & 0.0035 \\
M3 & Defect a - Healthy & 2200 & 60 & 0.017 \\
M4 & Defect b - Healthy & 3200 & 60 & 0.0091 \\
M5 & Defect b - Healthy & 2200 & 30 & 0.011 \\
M6 & Defect c - Healthy & 3100 & 40 & 0.015 \\
M7 & Defect c - Healthy & 3100 & 124 & 0.034 \\
M8 & Defect a - Healthy & 3100 & variable & 0.017 \\
M9 & Defect c - Healthy & 3100 & variable & 0.0024 \\
\hline
\end{tabular}



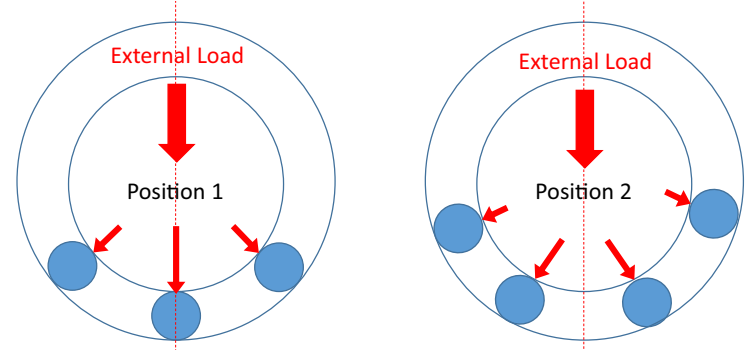

Fig. 7. Illustration of normal load distribution versus rolling element angular position.

These results lead to the following conclusions:

- The proposed tool is robust as it gives similar signals from different measurements.

- From measurements performed on a mechanical system with two identical bearings it is possible to have healthy information for each bearing.

- Speed variations associated to rotation of a healthy bearing are very weak ( $0.025 \%$ of the macroscopic speed of the shaft) but nevertheless they may be detected. Their shape is close to a sinusoid and sensitivity to load conditions validates the fluctuation of rolling element load during rotation as their origin.

\subsection{Analysis of measurements M2, M4 and M6: Influence of defect size}

Measurements M2 and M4 are performed for the same operating conditions (macroscopic speed $60 \mathrm{rad} / \mathrm{s}$, radial load $3200 \mathrm{~N}$ ), but with two different faulty bearings, the second bearing of the experimental setup being healthy. As seen before, indicator $\mathrm{H}_{\mathrm{sm}}$ allows the detection of a faulty bearing but not the estimation of the fault size as the smallest defect (a, measurement $M 2, H_{s m}=0.017$ ) gives an higher indicator level than the defect (b, measurement $M 4, H_{s m}=0.011$ ). Fig. 9 shows the filtered speed variations for each measurement and for faulty and healthy bearings.

From the two measurements, the presence of defect on bearing outer race largely modifies the shape of the filtered speed variations curve associated with the faulty bearing. This modification is characterized with a high magnitude peak. By using the healthy bearing curve (in dash point) and knowing the size of the defect expressed in percentage of the period $A_{\theta}$, we are able to conclude that this maximum is related to the defect exit. The entrance is more difficult to identify but it seems to be followed by a minima.

When outer races are changed on the experimental setup, they are theoretically mounted in a position such that the defect is located under the radial load. Therefore, by comparing healthy and faulty curves in Fig. 9a it is possible to associate extrema of the healthy curve with position of rolling elements (see Fig. 7) during rotation. Minimum of the sine wave is associated to position 1 and its maximum is with the position 2. In the case of M4 measurement (Fig. 9b), it appears that the defect entrance is slightly shifted from the minimum of the healthy curve, indicating that the defect position is shifted of
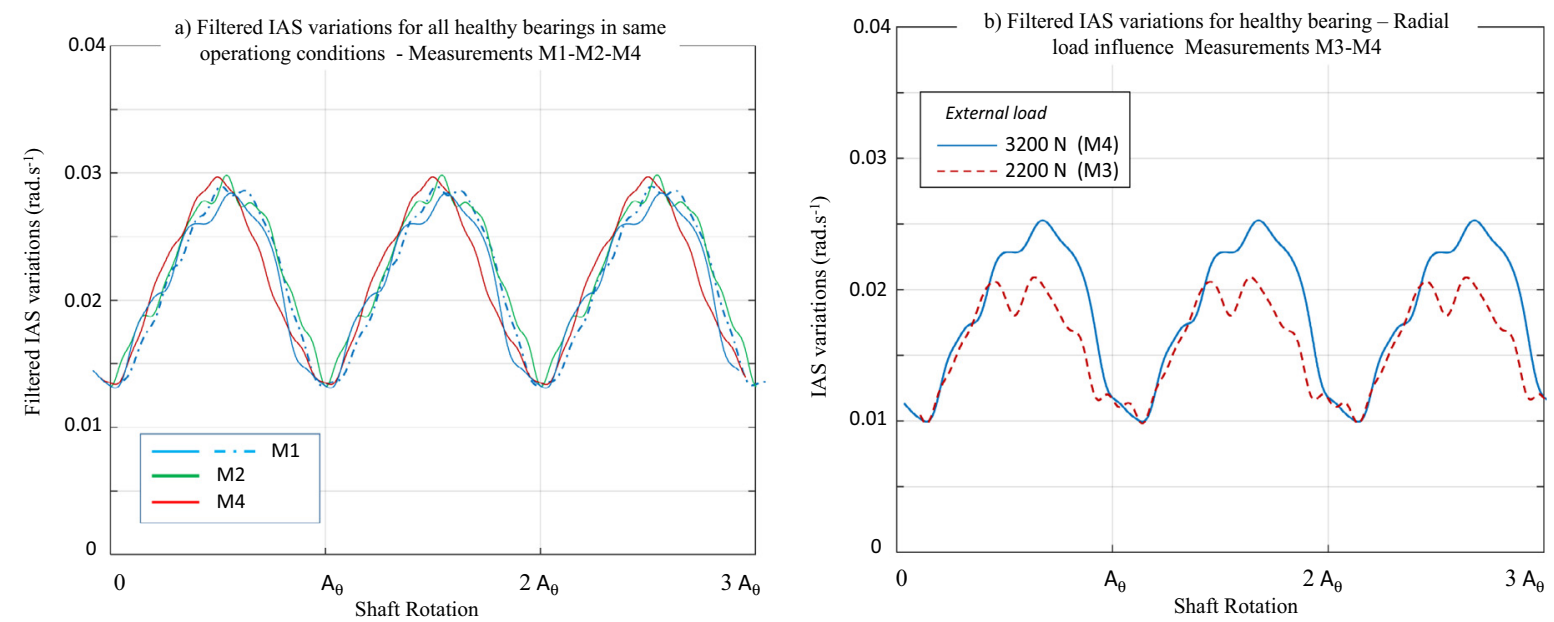

Fig. 8. Presence of BPOO components for different measurements and load conditions. 

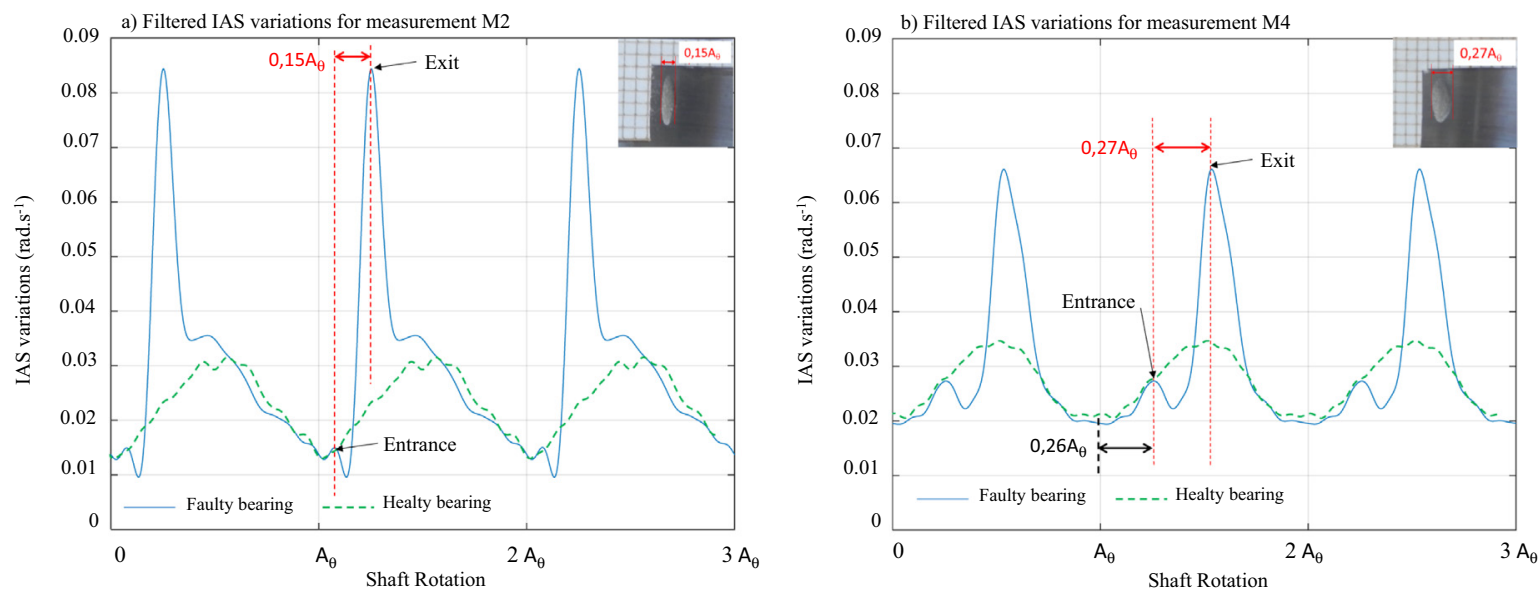

Fig. 9. Filtered IAS variation for healthy and faulty bearing working in the same operating condition - influence of defect size.

about $0.26 \mathrm{~A}_{\theta}$ or $5.5^{\circ}$ from the radial load direction. This position shift may also explain the reason why speed variations from M4 are weaker $\left(\mathrm{H}_{\mathrm{sm}}\right.$ indicator is lower) than those obtained from M2 measurement while the defect is larger. In that case, defect entrance appears easier to detect.

Fig. 10a shows filtered speed variations from the bearings of measurement M6. The defect (c) exhibits a large modification of speed variations with a magnitude of about $0.18 \mathrm{rad} / \mathrm{s}$ which is $0.45 \%$ of the nominal shaft speed ( $40 \mathrm{rad} / \mathrm{s}$ ).

The defect exit remains easy to detect and associated to the maxima of speed variations but a second extrema is also visible for different measurements. As reported in Fig. $10 \mathrm{~b}$, this second extrema is located at a distance of $0.33 \mathrm{~A}_{\mathrm{o}}$ from the entrance and could be related to the surface roughness and to the shape of the defect, more complex than for the other defects. It could be associated to the partial contact between the race and the rolling element.

The previous analysis has shown the advantages of the tool for estimating the size of the defect. In the speed variation signals the exit is clearly located as the entrance is more difficult to locate. In association with vibration analysis in which entrance looks easier to detect, the combination of these two signal analyses may offer a good alternative in defect size estimation. Other information like the defect location relative to the load direction or the shape of the defect are also available with IAS measurement but remain for further investigations.

\subsection{Analysis of measurements M2/M3 - M6/M7 and M4/M5 - M2/M7: Influence of operating conditions}

The aim of this section is to estimate the influence of operating conditions on the speed variations. Measurements M2 and M3 are performed for defect (a), for the same macroscopic rotating speed $\left(60 \mathrm{rad} \cdot \mathrm{s}^{-1}\right)$ but with different external radial load

a) Filtered IAS variations for measurement M6

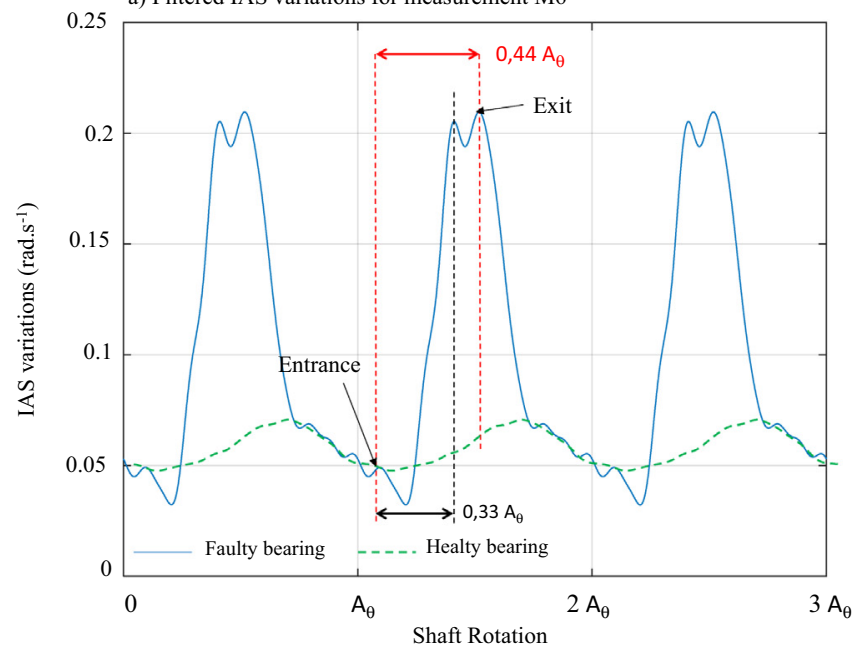

b) Defect (c) geometry

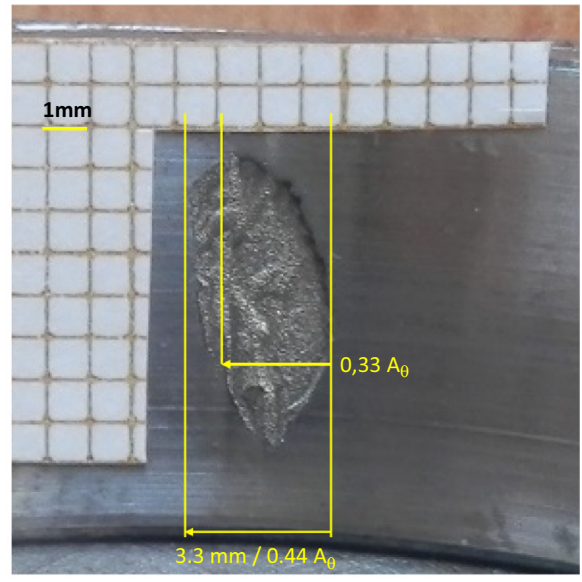

Fig. 10. Filtered IAS variation for healthy and faulty bearing for measurement M6 and corresponding defect geometry. 
( $3200 \mathrm{~N}$ and $2200 \mathrm{~N}$ ). Corresponding $\mathrm{H}_{\mathrm{sm}}$ indicators are 0.017 and 0.009. Measurement M6 and M7 are performed for defect (c), under the same external radial load $\left(3100 \mathrm{~N}\right.$ ) but with different macroscopic speed ( $40 \mathrm{rad} \cdot \mathrm{s}^{-1}$ and $\left.124 \mathrm{rad} \cdot \mathrm{s}^{-1}\right)$. Corresponding indicators $\mathrm{H}_{\mathrm{sm}}$ are 0.045 and 0.014 . For both case, evolution of the indicators may only confirm the influence of operating conditions on the detectability of the defect. Next figures present the filtered IAS variations associated with each case.

Operating conditions govern the magnitude of speed variations induced by defect but the general shape of the perturbation and particularly the locations of entrance and exit remain unchanged (the defect geometry remains unchanged). The analysis reports the following:

- An increase of the radial load leads to an increase of the perturbation magnitude, in the current case an increase of $45 \%$ of the load induces an increase of perturbation magnitude of $66 \%$.

- An increase of the speed leads to a decrease of the perturbation magnitude, in the current case an increase of $210 \%$ of the speed induces a decrease of perturbation magnitude of $55 \%$.

Effects of operating conditions on speed variations associated to bearing defects are obviously nonlinear.

In the case of defect (c), in Fig. 11b one has to notice that secondary maxima are detectable in both measurements in the same relative angular location. Therefore and as stated in the previous section, this phenomenon is mostly related to the defect geometry and not to the system dynamics.

Fig. 12a shows the results for measurements M4 and M5 with defect (b) mixing different load conditions (3200 N/2200 N) and speed conditions $\left(60 \mathrm{rad} \cdot \mathrm{s}^{-1} / 30 \mathrm{rad} \cdot \mathrm{s}^{-1}\right.$ ). In both configurations, the exit point is perfectly located and defect size may be estimated. From measurement M5 to measurement M4, shaft speed increases of $100 \%$ and load of $45 \%$ leading to a decrease of $36 \%$ of the magnitude of the perturbation.

Fig. 12b shows the results for measurements M2 (for defect (a)) and M7 (for defect (c)) both exhibiting the same $\mathrm{H}_{\text {sm }}$ level of 0.017 , the curve placement being insured by locating extrema associated to the defect exit. Despite the same magnitude of the speed variations of $0.07 \mathrm{rad} / \mathrm{s}$ and different operating conditions in speed, the proposed method allows to dissociate the changes in defect geometry.

Furthermore, $\mathrm{H}_{\mathrm{sm}}$ indicator appears not efficient in estimating defect size but it is representative of the magnitude of the speed variations induced by the defect as shown in Table 3.

To conclude, operating conditions characteristics and length of the defect are mixed in global indicators based on harmonics magnitude but separated in the proposed analysis.

\subsection{Analysis of measurements M8 and M9: Non stationary operating conditions}

This section is dedicated to the analysis of IAS in non-stationary conditions and results are shown in order to introduce some research perspectives about non slipping conditions.

Measurement M8 performed with defect (a) corresponds to very large and sharp speed variations as shown in Fig. 13a. Nevertheless, proposed approach can be applied. Resulting IAS variations are plotted in Fig. 13b for four different shaft angular positions. It appears that the proposed tool is not affected by the large speed variations and still allows identifying the presence of the defect and its length.

Curiously in the range $0-600 \mathrm{rev}$. where the speed is stable around $83 \mathrm{rad} \cdot \mathrm{s}^{-1}$, filtered speed variations (red curve) exhibit an oscillation not found for other speed conditions. This phenomenon is also present for other measurements operated at the
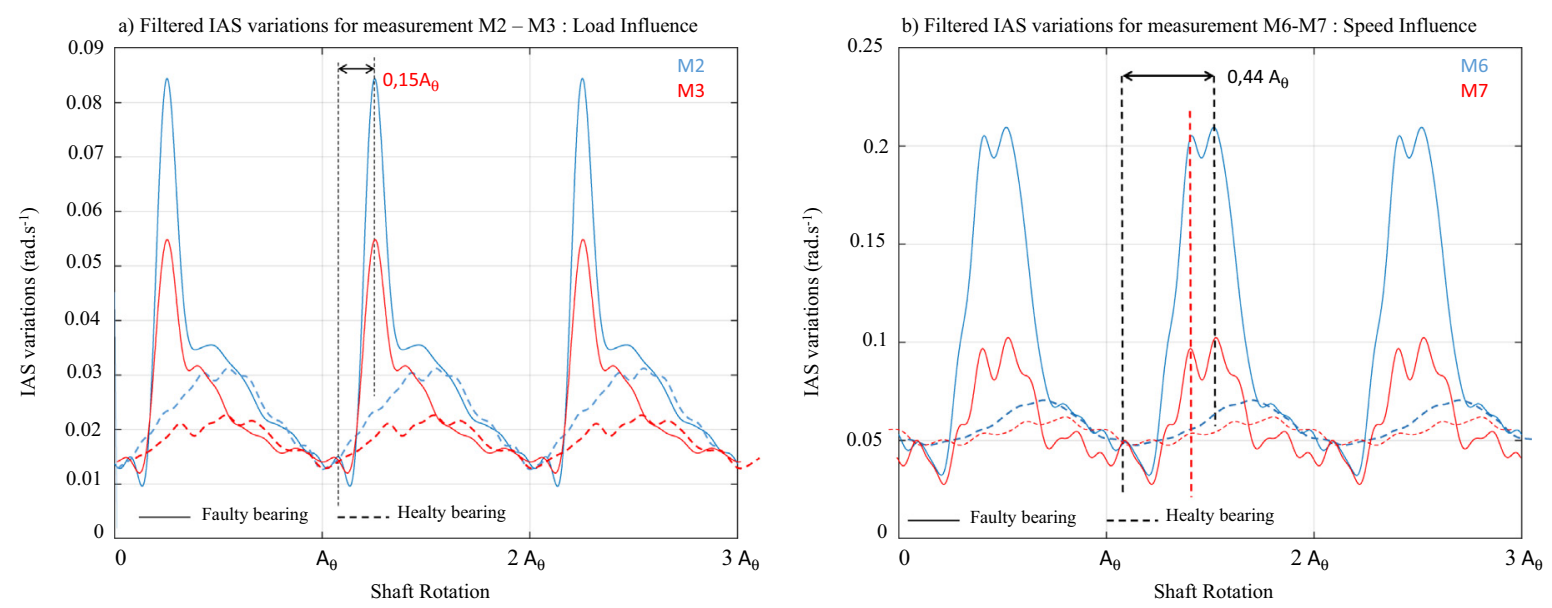

Fig. 11. Filtered I.A.S variations - Influence of load and speed. 

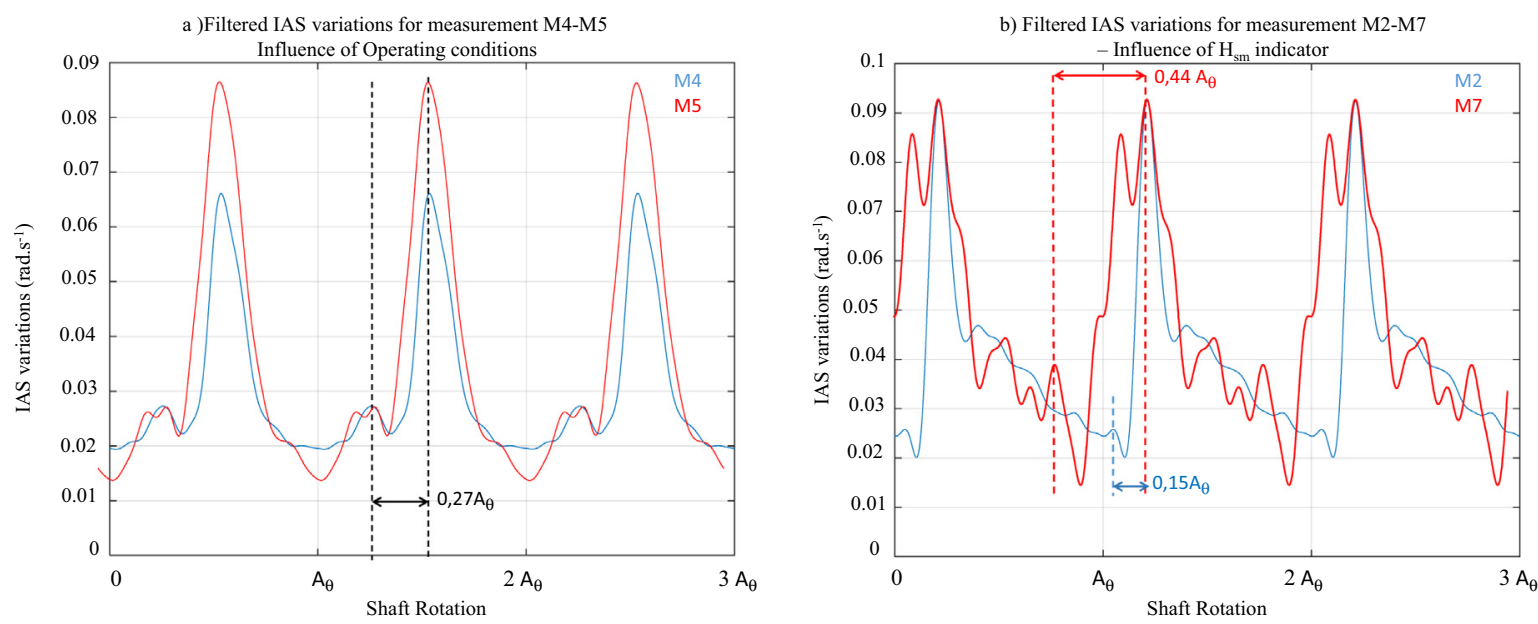

Fig. 12. Filtered I.A.S variations - Influence of operating conditions and $\mathrm{H}_{\mathrm{sm}}$ indicator.

Table 3

Global indicator $\mathrm{H}_{\mathrm{sm}}$ for bearing 1 and corresponding speed fluctuations.

\begin{tabular}{lll}
\hline Measurement & $\mathrm{H}_{\mathrm{sm} 1}$ & Speed variations $\left(\mathrm{rad} \cdot \mathrm{s}^{-1}\right)$ \\
\hline M1 & 0.0035 & 0.03 \\
M2 & 0.017 & 0.07 \\
M3 & 0.0091 & 0.04 \\
M4 & 0.011 & 0.045 \\
M5 & 0.015 & 0.06 \\
M6 & 0.034 & 0.15 \\
M7 & 0.017 & 0.07 \\
\hline
\end{tabular}

same speed condition ( $83 \mathrm{rad} \cdot \mathrm{s}^{-1}$ ) and may probably be linked to interaction between cyclic excitation and structural resonance.

Measurement M9 is performed with the defect (c) and corresponds to a speed run up as shown in Fig. 14a.

For this measurement, analysis is more difficult as the BPOO harmonic associated to the faulty bearing is not clearly visible in the angle frequency spectrum shown in Fig. 14b. Speed variations extracted from the whole measurement and filtered with a large frequency band containing all the BPOO components exhibit interesting results shown in Fig. 15. Using the whole signal, filtered IAS variations have been computed using the proposed tool. The red window in Fig. 14b is used. It

a) M8 macroscopic speed changes
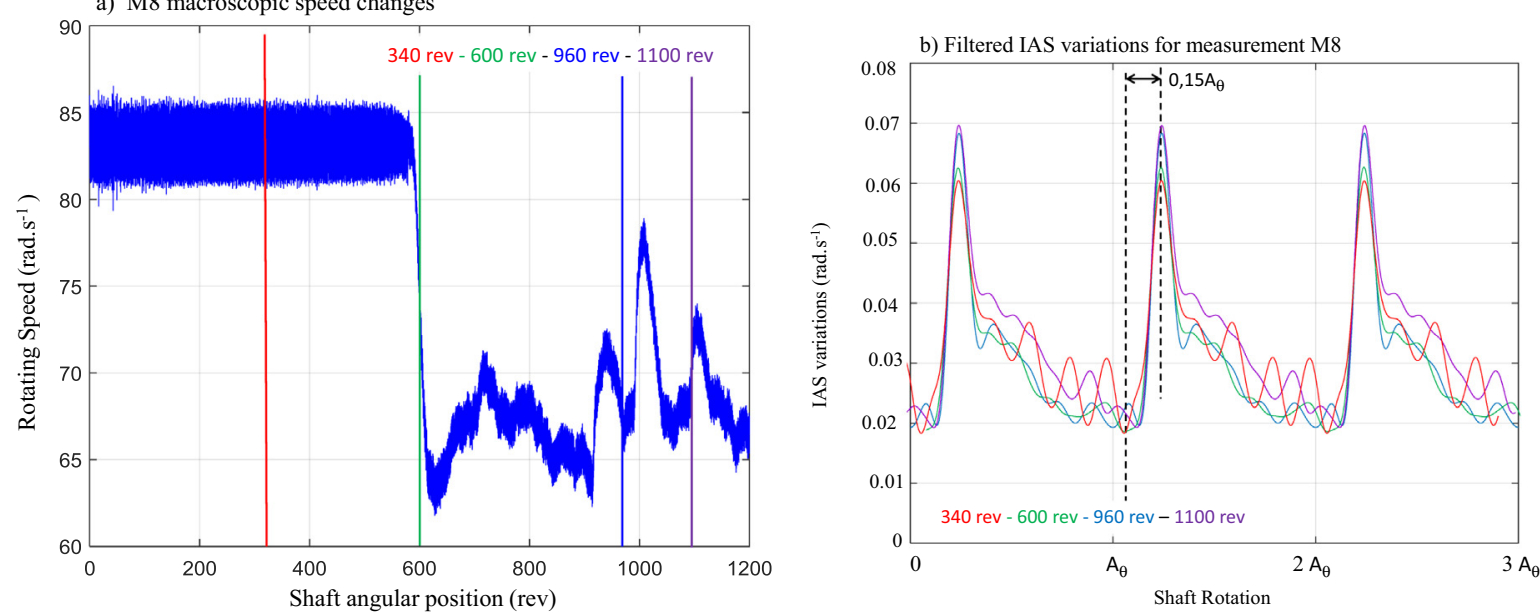

Fig. 13. Analysis of measurement M8 for faulty bearing (a) for non-stationary operating conditions. 
a) M9 macroscopic speed changes

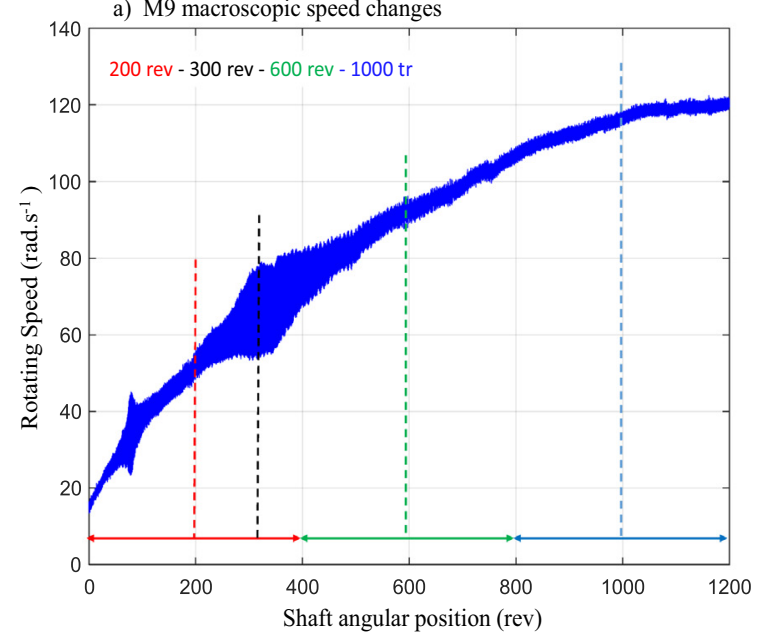

b) Speed variations spectrum for mesure M9 - Zoom around BPOO

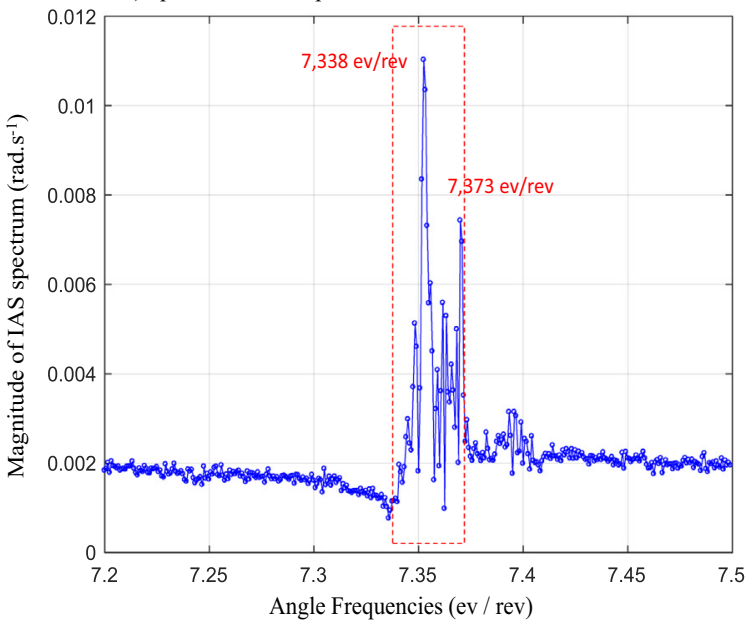

Fig. 14. Analysis of measurement M9 for faulty bearing (c) for non-stationary operating conditions.

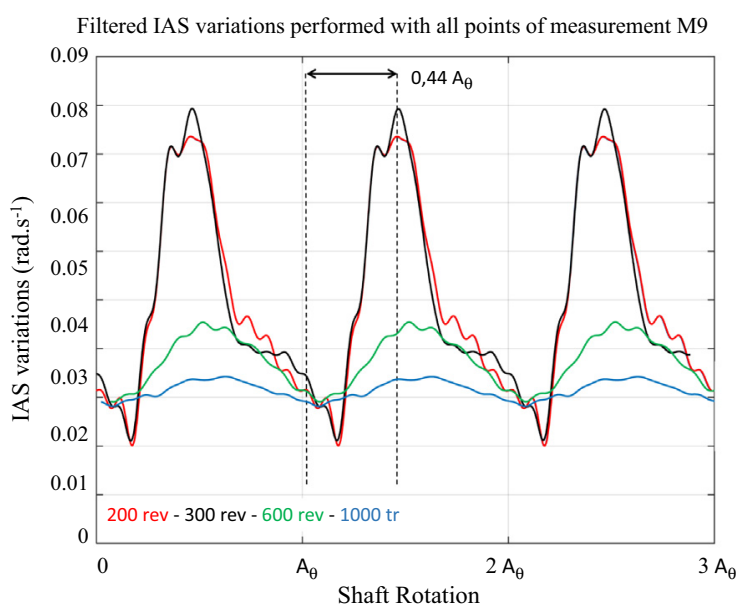

Fig. 15. Filtered signal at four locations for measurement M9 for faulty bearing (c) in non-stationary operating conditions.

is centered on $7.35 \mathrm{ev} / \mathrm{rev}$ and have a length of 41 channels $(n=20)$. Then zooms with a length of $3 \mathrm{~A}_{\theta}$ are performed around different angular positions and plotted in Fig. 15. At first glance, it could be seen that the proposed tool does not allow reconstructing the disturbance due to the defect on the whole measurement. In fact, in the first part of the filtered signal (200 rev and $300 \mathrm{rev}$ ), effects of the faulty bearing are correctly reconstructed. The shape of disturbance is the same as the one obtained with this defect (measurements M6-M7), characteristic extrema attesting their link to the defect geometry. In the second part of the signal (600 rev and $1000 \mathrm{rev}$ ) the frequency filter is located closer to the healthy bearing BPOO and exhibits the effects of this bearing rather than the faulty one.

For further investigations, measurement M9 offering 1200 shaft revolutions is divided in 3 parts of length 400 revolutions as indicated in Fig. 14a. Spectra extracted from each part are shown in Fig. 16a indicating that a sliding of the BPOO may occur and may be detected in measurement M9 with lower resolution due to shorter length of analysis. This phenomenon is properly at the origin of the fact that effects of faulty bearing are not correctly reconstructed in the previous analysis. For a correct use of the proposed method, one has to ensure that the BPOO harmonics must be in the filter order bands on the whole duration of the measurement. By applying the reconstruction tool with three filters defined for each part with similar bandwidths, it is possible to better reconstruct the shape of the faulty bearing all along the measurement (see Fig. 16b).

Results shown in this section clearly exhibit that the tool may be used in non stationary conditions without modification if there is no slippage in bearing of interest. When BPOO is slightly changing during measurement, one has to take particular care in defining correctly the filter. The proposed approach may be improved by using further filtering methods in order to tackle resolution issues in spectrum editing procedure or to implement tracking filters. 



Fig. 16. Analysis of measurement M9 for faulty bearing (c) for non-stationary operating condition analysis performed by splitting the initial measurement made of 6000000 points in 3 areas of 2000000 points.

\section{Conclusion}

The work presented in this paper is based on previous results which have shown the interest of the angular approach regarding the analysis of vibration and Instantaneous Angular Speed signals for monitoring rotating machines. These studies have shown that the effect of a faulty bearing on the rotating speed may be detectable by simple spectral analysis of the Instantaneous Angular Speed if sampled in the angular domain over a large number of points. These facts being established, the measurements performed on complex devices do not allow to clearly identify the relationships between operating conditions and effects of faults on the rotating speed of the machine and do not allow the estimation of the magnitude and the shape of the resulting disturbances. To gain a better understanding of these phenomena a signal processing tool is proposed essentially based on the use of a filter defined in the angular frequency domain.

Using this tool to actual signals from a test bench has allowed to quantify the magnitude of the speed variations related to the BPOO (Ball Pass Order on the Outer race) of the bearings under monitoring. For healthy bearing this magnitude is about $0.01 \%$ of the macroscopic speed and the shape of the speed variation is close to a sinusoid, mainly due to the load sharing between rollers. For faulty bearings and for the considered operating conditions, magnitude rises up to $0.1 \%$ and the speed variations present a specific shape that can be correlated to the size of the defect. This tool also distinguishes the speed variations due to an evolution of the defect to those due to variations in operating conditions. It can also explain why the detectability of a defect may depend on the operating conditions. In classical frequency indicators based on harmonics magnitude, modifications due to operating conditions and length of the defect are generally mixed. On the opposite in the analysis proposed herein, these two contributions are clearly separated. Another interesting point is that the proposed tool can be used in stationary or non-stationary operating conditions and leads to the same results under the condition of no slipping phenomenon in the bearing kinematics. Some results in non-stationary conditions have been presented when BPOO is spread over several order components during measurement mainly due to slipping. The proposed interpretation based on this single measurement must be further investigated with more adapted signal processing tools.

Applied to 9 measurements and 4 levels of tapered roller bearing defects, the proposed approach seems very promising but it must still be improved and tested on other measurements, other bearings and other setups. These experimental results should be compared to those obtained with phenomenological models in order to understand the transfer path between the fault and its manifestation. Another perspective lies on extending this approach to inner bearing defect as the modulations due to the rotation of the shaft have to be taken into account.

\section{Acknowledgements}

Authors gratefully acknowledge the support of LaMCoS Laboratory and Maia-Eolis for partially supporting this research activity.

\section{References}

[1] R.B. Randall, J. Antoni, Rolling element bearing diagnostics - a tutorial, Mech. Syst. Sig. Process. 25 (2) (2011) 485-520.

[2] A.M. Al-Ghamd, D. Mba, A comparative experimental study on the use of acoustic emission and vibration analysis for bearing defect identification and estimation of defect size, Mech. Syst. Sig. Process. 20 (7) (2006) 1537-1571. 
[3] D. Rémond, J. Antoni, R.B. Randall, Editorial for the special issue on Instantaneous Angular Speed (IAS) processing and angular applications, Mech. Syst. Sig. Process. 44 (1-2) (2014) 1-4.

[4] H. André, A. Bourdon, D. Rémond, Instantaneous Angular Speed monitoring of a 2MW wind turbine using a parametrization process, Proceedings of the International Conference on Condition Monitoring of Machinery in Non-Stationary Operations, International Conference on Condition Monitoring of Machinery in Non-Stationary Operations, 26-28 March 2012, Hammamet, Tunisia, 2012.

[5] J.L. Gomez, A. Bourdon, H. André, D. Rémond, Modelling deep groove ball bearing localized defects inducing instantaneous angular speed variations, Tribol. Int. 98 (2016) 270-281.

[6] N. Sawalhi, R.B. Randall, Simulation of the vibration generated by entry and exit to/from a spall in a rolling element bearing, Proceedings of 20 th International Congress on Acoustics, ICA 2010, 23-27 August 2010, Sydney, Australia, 2010.

[7] F. Bonnardot, M. El Badaoui, R. Randall, J. Daniere, F. Guillet, Use of the acceleration of a gearbox in order to perform angular resampling (with limited speed fluctuation), Mech. Syst. Sig. Process. 19 (4) (2005) 766-785.

[8] J. Antoni, R.B. Randall, On the use of the cyclic power spectrum in rolling element bearings diagnostics, J. Sound Vib. 281 (2) (2005) $463-468$.

[9] Y. Jianguo, P. Lijun, W. Zhihua, Z. Yichen, Y. Xinping, Fault detection in a diesel engine by analysing the instantaneous angular speed, Mech. Syst. Sig. Process. 15 (3) (2001) 549-564.

[10] L. Yuhua, F. Gu, G. Harris, A. Ball, N. Benett, K. Travis, The measurement of instantaneous angular speed, Mech. Syst. Sig. Process. 19 (4) (2005) 786-805.

[11] S. Yu, X. Zhang, A data processing method for determining instantaneous angular speed and acceleration of crankshaft in an aircraft engine-propeller system using a magnetic encoder, Mech. Syst. Sig. Process. 24 (4) (2010) 1032-1048.

[12] L. Renaudin, F. Bonnardot, O. Musy, J.B. Doray, D. Rémond, Natural roller bearing fault detection by angular measurement of true instantaneous angular speed, Mech. Syst. Sig. Process. 24 (7) (2010) 1998-2011.

[13] L.D. Meyer, F.F. Ahlgren, B. Weichbrodt, An analytic model for ball bearing vibrations to predict vibration response to distributed defects, J. Mech. Des. $102(2)(1980) 205-210$

[14] N. Sawalhi, R.B. Randall, Vibration response of spalled rolling element bearings: Observations, simulations and signal processing techniques to track the spall size, Mech. Syst. Sig. Process. 25 (2011) 846-870.

[15] J. Antoni, R.B. Randall, The spectral kurtosis: application to the vibratory surveillance and diagnostics of rotating machines, Mech. Syst. Sig. Process. 20 (2) (2006) 308-331.

[16] F. Gu, I. Yesilyurt, L. Yuhua, G. Harris, A. Ball, An investigation of the effects of measurement noise in the use of instantaneous angular speed for machine diagnosis, Mech. Syst. Sig. Process. 20 (6) (2006) 1444-1460.

[17] H. André, J. Antoni, Z. Daher, D. Rémond, Comparison between angular sampling and angular resampling methods applied on the vibration monitoring of a gear meshing in non-stationary conditions, 'Proceedings of the International Conference on Noise and Vibration Engineering', Leuven, Belgium, September 20-22, 2010.

[18] H. André, A. Bourdon, J. Antoni, D. Rémond, Precision of the IAS monitoring system based on the elapsed time method in the spectral domain, Proceedings of the International Conference on Noise and Vibration Engineering, ISMA 2012, 17-19 September 2012, Leuven, Belgium, 2012.

[19] A. Bourdon, H. André, D. Rémond, Numerical investigations on the magnitude of disturbance that could be detected through IAS, Proceedings of the International Conference on Condition Monitoring of Machinery in Non-Stationary Operations, International Conference on Condition Monitoring of Machinery in Non-Stationary Operations, 26-28 March 2012, Hammamet, Tunisia, 2012.

[20] H. André, F. Girardin, A. Bourdon, J. Antoni, D. Rémond, Precision of the IAS monitoring system based on the elapsed time method in the spectral domain', Mech. Syst. Sig. Process. 44 (1-2) (2014) 14-30.

[21] A. Bourdon, D. Remond, S. Chesne, H. Andre, Reconstruction of the instantaneous angular speed variations caused by a spall defect on a rolling bearing outer ring correlated with the length of the defect, in: G. Dalpiaz (Ed.), Advances in Condition Monitoring of Machinery in Non-Stationary Operations. Lecture Notes in Mechanical Engineering, Springer, Berlin, Heidelberg, 2014. 